Research Paper

\title{
The Versatile Use of Temporoparietal Fascial Flap
}

\section{Cenk Demirdover ${ }^{1 凶}$, Baris Sahin ${ }^{2}$, Haluk Vayvada ${ }^{1}$, Hasan Yucel Oztan ${ }^{3}$}

1. Department of Plastic Reconstructive and Aesthetic Surgery, Dokuz Eylul University, Faculty of Medicine, Izmir, TURKEY.

2. Clinic of Plastic Reconstructive and Aesthetic Surgery, Public Hospital, Mus, Turkey.

3. Clinic of Plastic Reconstructive and Aesthetic Surgery, Izmir Atatürk Education and Research Hospital, Izmir, TURKEY.

Corresponding author: Cenk Demirdover, Department of Plastic Reconstructive and Aesthetic Surgery, Dokuz Eylul University, Faculty of Medicine, Izmir, TURKEY. Phone: +90 5324246269 E-mail: cenk.demirdover@deu.edu.tr or cenkddr@gmail.com

(c) Ivyspring International Publisher. This is an open-access article distributed under the terms of the Creative Commons License (http://creativecommons.org/ licenses/by-nc-nd/3.0/). Reproduction is permitted for personal, noncommercial use, provided that the article is in whole, unmodified, and properly cited.

Received: 2011.04.12; Accepted: 2011.05.31; Published: 2011.06.10

\begin{abstract}
Background: The pedicled or free temporoparietal fascial has been used in many areas, especially in head and neck reconstruction. This thin, pliable, highly vascularized flap may be also transferred as a carrier of subjacent bone or overlying skin.

Objective: The aim of this study is to report our experience in versatile use of temporoparietal fascial flap (TPFF) and discuss the surgical anatomy and technique.

Patients and Methods: A total number of $57 \mathrm{TPFF}$ have been used in periorbital, mid-facial, auricular, and tracheal reconstruction due to tumor resection, trauma, and congenital ear deformities.

Results: All the flaps were successfully transferred without any major complication. The cosmetic results were quite satisfactory to all patients.

Conclusion: The advantages and minimal donor site morbidity of TPPF makes this flap a good choice in many reconstructive procedures.
\end{abstract}

Key words: temporoparietal fascial flap, superficial temporal fascia, head and neck reconstruction

\section{Introduction}

The temporoparietal fascial flap (TPFF) has been used as a pedicled, free, or composite flap with calvarial bone or hair-bearing skin in reconstruction of the defects of periorbital, mid-facial, auricular regions, and extremities. ${ }^{1-3}$ This thin, pliable, and highly vascularized flap, accepts skin grafts on both sides. Its anatomical proximity and minimal donor site morbidity provides a good option for the reconstructive surgeons. Since Brent et al reported secondary ear reconstruction with cartilage grafts covered by axial, random and free flaps of temporoparietal fascia, anatomical researches of temporal area gained popularity. ${ }^{4}$ When its advantages are combined with the surgeon's imagination, many treatment options can be created in reconstruction of the defects due to trauma, tumor resection, congenital deformities, and radiation treatment.

In this study, relevant surgical anatomy, technical aspects, the flap design and its versatile use, patient selection and evaluation, and our results in 57 patients are discussed.

\section{Patients and Methods}

Between 2003 and 2010, the TPFF has been used in reconstruction of various defects of 57 patients (Table 1$)$. Thirty two were $(56.1 \%)$ males and 15 females $(43.9 \%)$, with an average age of 51.3 years (range, 18-85 years). The TPFF was used in 46 patients 
$(80.7 \%)$ for head and neck reconstruction after tumor resection; in 5 patients $(8.7 \%)$ for congenital ear deformities; and in 6 patients $(10.6 \%)$ for posttraumatic reconstruction. Periorbital, malar, auricular and tracheal areas were reconstructed.

Table 1. The data showing the age, sex, etiologic factors, tumor type, follow-up time, and complications of the patients.

\begin{tabular}{|c|c|c|c|c|c|c|}
\hline Patient & Age & Sex & Etiology & Localization & Follow-up & Complication \\
\hline 1 & 18 & $\mathrm{~F}$ & Congenital ear deformity & Ear & 17 & None \\
\hline 2 & 28 & $\mathrm{~F}$ & Congenital ear deformity & Ear & 14 & Incisional alopecia \\
\hline 3 & 27 & M & Congenital ear deformity & Ear & 23 & None \\
\hline 4 & 25 & M & Congenital ear deformity & Ear & 14 & None \\
\hline 5 & 27 & M & Congenital ear deformity & Ear & 20 & None \\
\hline 6 & 39 & M & Facial Trauma & Cheek & 8 & None \\
\hline 7 & 34 & M & Facial Trauma & Mid-face & 42 & None \\
\hline 8 & 34 & M & Facial Trauma & Cheek & 21 & None \\
\hline 9 & 17 & M & Tracheal defect & Trachea & 39 & None \\
\hline 10 & 29 & M & Traumatic ear deformity & Ear & 21 & None \\
\hline 11 & 29 & M & Traumatic ear deformity & Ear & 33 & Incisional alopecia \\
\hline 12 & 57 & $\mathrm{~F}$ & Tumor (SCC) & Orbit & 27 & None \\
\hline 13 & 66 & $\mathrm{~F}$ & Tumor (SCC) & Orbit & 9 & None \\
\hline 14 & 85 & $\mathrm{~F}$ & Tumor (BCC) & Orbit & 8 & None \\
\hline 15 & 69 & $\mathrm{~F}$ & Tumor (SCC) & Cheek & 19 & None \\
\hline 15 & 46 & $\mathrm{~F}$ & Tumor (SCC) & Orbit & 14 & None \\
\hline 17 & 58 & $\mathrm{~F}$ & Tumor (SCC) & Orbit & 29 & None \\
\hline 18 & 47 & $\mathrm{~F}$ & Tumor (MM) & Orbit & 17 & None \\
\hline 19 & 41 & $\mathrm{~F}$ & Tumor (SCC) & Orbit & 58 & None \\
\hline 20 & 66 & $\mathrm{~F}$ & Tumor (SCC) & Orbit & 8 & Incisional alopecia \\
\hline 21 & 44 & $\mathrm{~F}$ & Tumor (SCC) & Orbit & 18 & Hematoma \\
\hline 22 & 71 & $\mathrm{~F}$ & Tumor (SCC) & Orbit & 10 & None \\
\hline 23 & 65 & $\mathrm{~F}$ & Tumor (SCC) & Orbit & 9 & None \\
\hline 24 & 50 & $\mathrm{~F}$ & Tumor (MM) & Mid-face & 24 & None \\
\hline 25 & 25 & M & Tumor (BCC) & Orbit & 9 & None \\
\hline 26 & 33 & M & Tumor (MM) & Orbit & 11 & None \\
\hline 27 & 58 & M & Tumor (SCC) & Orbit & 7 & None \\
\hline 28 & 49 & M & Tumor (SCC) & Orbit & 14 & None \\
\hline 29 & 21 & M & Tumor (SCC) & Orbit & 31 & None \\
\hline 30 & 63 & M & Tumor (SCC) & Orbit & 23 & None \\
\hline 31 & 78 & M & Tumor (BCC) & Orbit & 27 & Incisional alopecia \\
\hline 32 & 29 & M & Tumor (SCC) & Orbit & 14 & None \\
\hline 33 & 47 & M & Tumor (SCC) & Orbit & 22 & None \\
\hline 34 & 54 & M & Tumor (SCC) & Orbit & 25 & None \\
\hline 35 & 71 & M & Tumor (SCC) & Orbit & 17 & None \\
\hline 36 & 57 & M & Tumor (BCC) & Orbit & 36 & None \\
\hline 37 & 57 & M & Tumor (MM) & Orbit & 18 & Hematoma \\
\hline 38 & 68 & M & Tumor (SCC) & Orbit & 26 & None \\
\hline 39 & 65 & M & Tumor (SCC) & Orbit & 15 & None \\
\hline 40 & 46 & M & Tumor (SCC) & Orbit & 50 & None \\
\hline 41 & 73 & M & Tumor (BCC) & Orbit & 27 & None \\
\hline 42 & 45 & M & Tumor (SCC) & Orbit & 42 & None \\
\hline 43 & 63 & M & Tumor (BCC) & Orbit & 34 & None \\
\hline 44 & 59 & M & Tumor (BCC) & Orbit & 25 & None \\
\hline 45 & 58 & M & Tumor (SCC) & Mid-face & 18 & None \\
\hline
\end{tabular}




\begin{tabular}{|c|c|c|c|c|c|c|}
\hline 46 & 38 & M & Tumor (SCC) & Orbit & 35 & None \\
\hline 47 & 45 & M & Tumor (MM) & Orbit & 12 & None \\
\hline 48 & 67 & M & Tumor (SCC) & Orbit & 25 & None \\
\hline 49 & 70 & M & Tumor (BCC) & Orbit & 11 & None \\
\hline 50 & 66 & M & Tumor (SCC) & Orbit & 13 & None \\
\hline 51 & 58 & M & Tumor (SCC) & Orbit & 17 & None \\
\hline 52 & 57 & M & Tumor (SCC) & Orbit & 24 & None \\
\hline 53 & 66 & M & Tumor (SCC) & Mid-face & 19 & Incisional alopecia \\
\hline 54 & 70 & M & Tumor (BCC) & Orbit & 10 & None \\
\hline 55 & 72 & M & Tumor (SCC) & Orbit & 9 & None \\
\hline 56 & 74 & M & Tumor (SCC) & Orbit & 6 & None \\
\hline 57 & 52 & M & Tumor (SCC) & Orbit & 21 & None \\
\hline
\end{tabular}

SCC: Squamous cell carcinoma, BCC: Basal cell carcinoma, MM: Malignant melanoma.

\section{Surgical Anatomy}

The superior temporal line, the frontal process of the zygomatic bone, and the zygomatic arch constitute superior, anterior, and inferior borders of the temporal fossa. Because it contains the temporalis muscle and its fasciae, temporal vessels, the temporal branch of the facial nerve, and the auriculotemporal nerve, it becomes more important in surgical dissection of this region.

Various names, such as temporoparietal fascia (TPF), superficial temporal fascia, epicranial aponeurosis, and galeal extension, have been used in order to define the fascial layers of the temporal region. ${ }^{5-6}$ All these names reflect an anatomical feature of the related fascia.

The TPF lies just beneath the hair follicles and subcutaneous fat of the temporal region. This fascia is attached superiorly to the superior temporal line and inferiorly to the lateral and medial surfaces of the zygomatic arch. ${ }^{7}$ The TPF must be distinguished from the denser and anatomically deeper temporalis fascia, which invests the temporalis muscle. ${ }^{8}$ The TPF is a 2-mm to 4-mm-thick layer of connective tissue which lies in the same plane with superficial muscular aponeurotic system (SMAS) and extends to the parietal region. ${ }^{5}$ Temporal and superficial temporal fascias are fused in the superior temporal line and both are attached at the level of zygoma. ${ }^{5-9}$ Loose areolar tissue exists between these two fascias. ${ }^{5,7}$

The superficial temporal artery which is the terminal branch of the external carotid artery runs within the TPF and supplies this fascia. Approximately $2-4 \mathrm{~cm}$ superior to the zygomatic arch artery divides frontal and parietal branches. The frontal branch is the terminal branch of the superficial temporal artery. The frontal and parietal branches are similar in size and frontal branch is less variable than parietal branch.9,10 The temporal vessels are located deeper at the level of the ear and approximately $10 \mathrm{~cm}$ above the crus helix, they take a more superficial course entering the subdermal plexus. ${ }^{8}$

The anatomic layers of the temporal region are shown in the Figure 1.

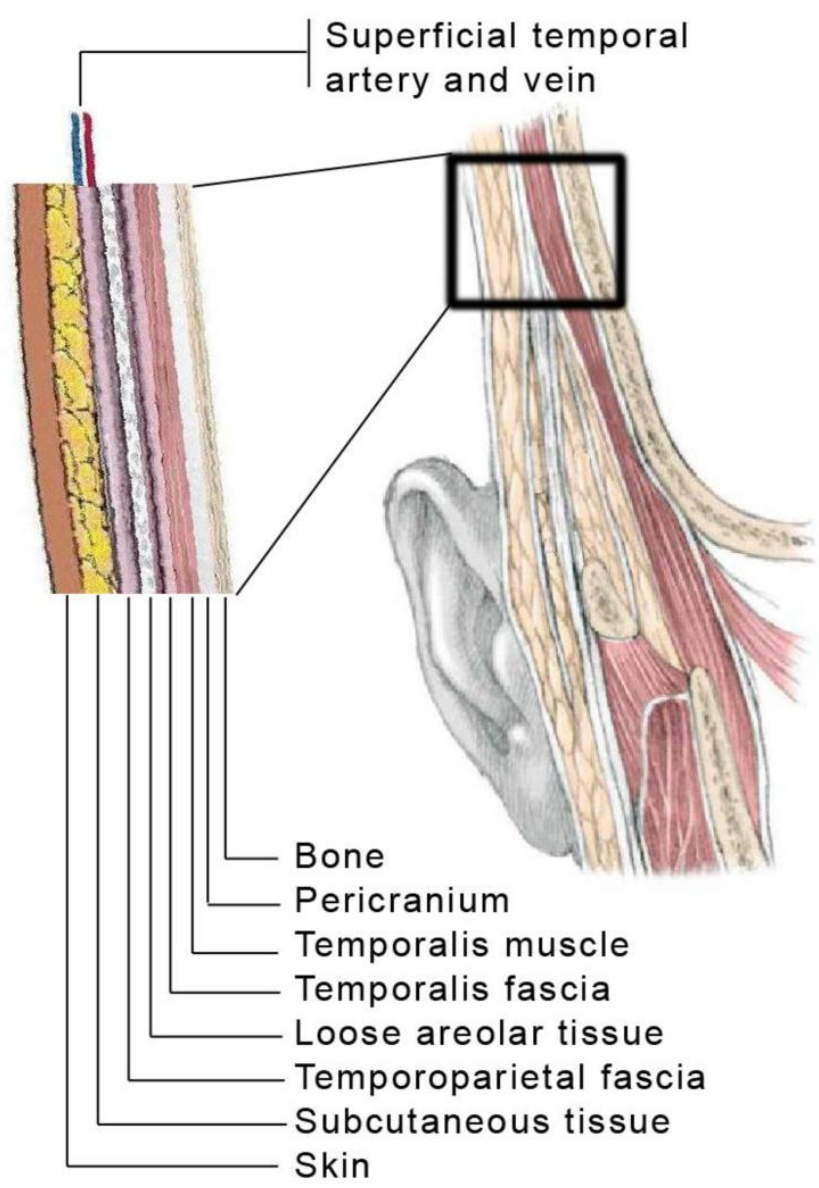

Figure 1: Anatomic layers of the temporal region. Note that superficial temporal artery and vein run within the temporoparietal fascia. 


\section{Surgical Technique}

Depending on the site that will be covered or reconstructed, different surgical approaches may be used. When a conventional fascial flap operation is planned, superficial temporal vessels in the pretragal region are palpated and the course of vessels is marked prior to incision. In order to locate the pedicle, hand-held Doppler device is also helpful. Entire scalp and the face are prepared with antiseptic solution. Some surgeons may prefer to shave the scalp's hair however we have only shaved the incision line.

Several incisions, such as lazy $\mathrm{S}$, inverted $\mathrm{T}$, Y-shaped, or zigzag incisions can be used. The incision is made starting from the preauricular region extending to the superior temporal line. This incision should be made carefully just over the temporal vessels. The superficial temporal fascia is dissected sharply with scalpel just beneath the hair follicles. Since there is no avascular plane between the skin and the fascia, a meticulous dissection should be carried out.

When the incision is completed, anterior and posterior scalp skin should be dissected. When adequate exposure is obtained, a proper flap and its axis of rotation are marked. At least $2-3 \mathrm{~cm}$ of tissue should be preserved around the pedicle at the pretragal level. The flap may be up to 14 to $17 \mathrm{~cm}$ in height and $10 \mathrm{~cm}$ in width ${ }^{11}$ The conventional fascial flap can be extended up to $3-4 \mathrm{~cm}$ superior to the origin of the temporal muscle. Then, the TPF is elevated from the deep temporal fascia by blunt dissection. If lengthening of the pedicle is needed, proximally superficial temporal vessels should be dissected cautiously in the pretragal region. Loupe magnification can be used during this procedure.

A fine-tipped bipolar electrocautery should be used carefully in hemostasis to avoid damaging hair follicles. After the TPF is transferred to the recipient site, hemovac drains are inserted and the donor site is sutured using 3/0 polypropylene. Depending on the amount of drainage, the drain is usually removed on the first or second postoperative day. An informed consent explaining all the details and possible complications should be obtained from all patients preoperatively.

Alopecia is the most common complication of this flap. Hematoma formation may occur if meticulous attention has not made for hemostasis. Partial or total flap loss may also be seen depending on inappropriate technique or previous surgery, irradiation, or carotid occlusion. When elevating anterior scalp flap, a particular care must be given to preserve the frontal branch of the facial nerve, otherwise, partial or total nerve injury may be seen.

\section{Patient reports}

\section{Patient 1}

A 71-year old male with a history of penetrating trauma, admitted to Ophthalmology clinic for slowly growing mass on his left eye (Figure 2 and 3). Two years after the surgical removal of the mass, he developed another mass originated from the conjunctiva. He was referred to our clinic after the incisional biopsy revealed poorly-differentiated squamous cell carcinoma. The tumoral mass as well as orbital contents were surgically removed and the orbit was reconstructed with a left TPFF and split-thickness skin graft (Figure 4). No complication was seen. In a two-year follow-up, he had no sign of recurrence.

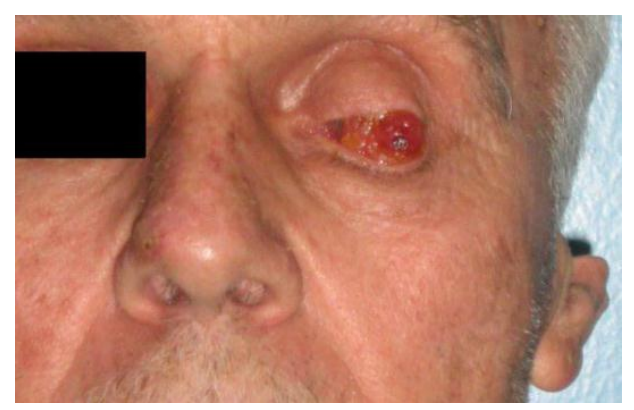

Figure 2: A 71-year old male with a slowly growing mass on his left eye. The biopsy revealed poorly-differentiated squamous cell carcinoma (Patient 1, preoperative frontal view).

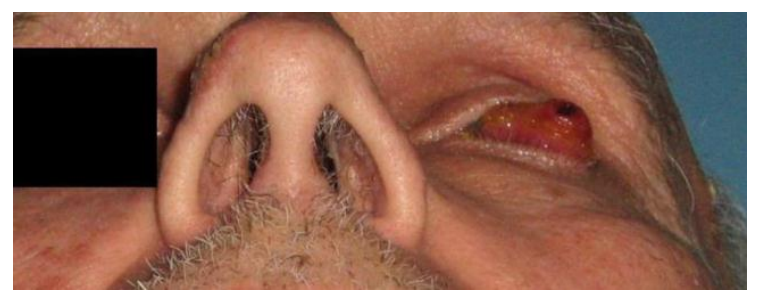

Figure 3: Preoperative basal view of the same patient (Patient 1).

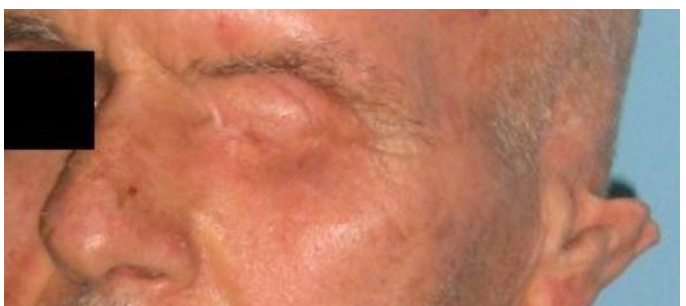

Figure 4: Orbital reconstruction was performed with a left TPFF and split-thickness skin graft. The amputation of the upper half of the left ear is not associated with this situation (Patient 1, postoperative oblique view). 


\section{Patient 2}

An 18-year old male admitted to an Emergency Department due to a car crash accident. He was followed-up in an Intensive Care Unit, requiring prolonged intubation. The cuff of the intubation tube resulted with necrosis at the central part of the larynx. Ear Nose Throat surgeons attempted to reconstruct the defect with local flaps which ended up with failure. He was referred to our clinic for the reconstruction of the $2 \times 2 \mathrm{~cm}$ of laryngeal defect (Figure 5). The major complaints were dysphonia, dyspnea, and wheezing. We have planned a two-stage reconstruction. At the first stage, rib cartilage graft was prefabricated within the TPF. Two weeks later, the microvascular transfer of the free TPFF was performed (Figure 6). The superior thyroid artery and vein were used as the recipient vessels. The patient was satisfied from the outcome of the surgery and at the seven years' follow-up he had no complaints about dysphonia or breathing problems (Figure 7).

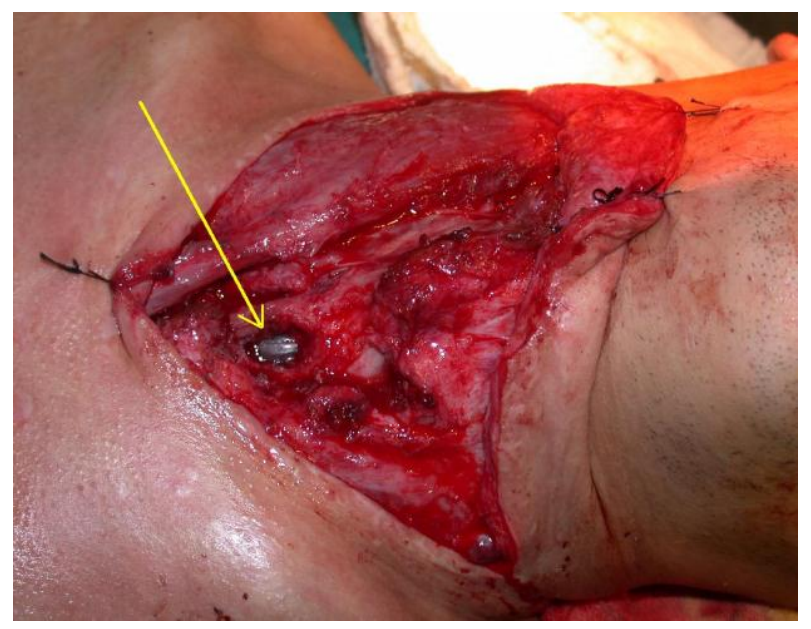

Figure 5: An 18-year old male with $2 \times 2 \mathrm{~cm}$ of laryngeal defect due to prolonged intubation. The arrow shows the defect and the intubation tube (Patient 2, intraoperative view).

\section{Results}

The TPFF has been used in reconstruction of various defects of 57 patients. Each of them was assessed in terms of age, sex, etiologic factors, tumor type, follow-up time, and complications (Table 1). Most of the patients were males (56.1\%) with an average age of 52.3 years (range, $18-85$ years). The TPFF was mainly used for head and neck reconstruction after tumor resection (46 patients, $80.7 \%$ ). In the rest of the patients, trauma (6 patients, $10.5 \%$ ) and congenital ear deformities (5 patients, $8.7 \%$ ) were the other etio-

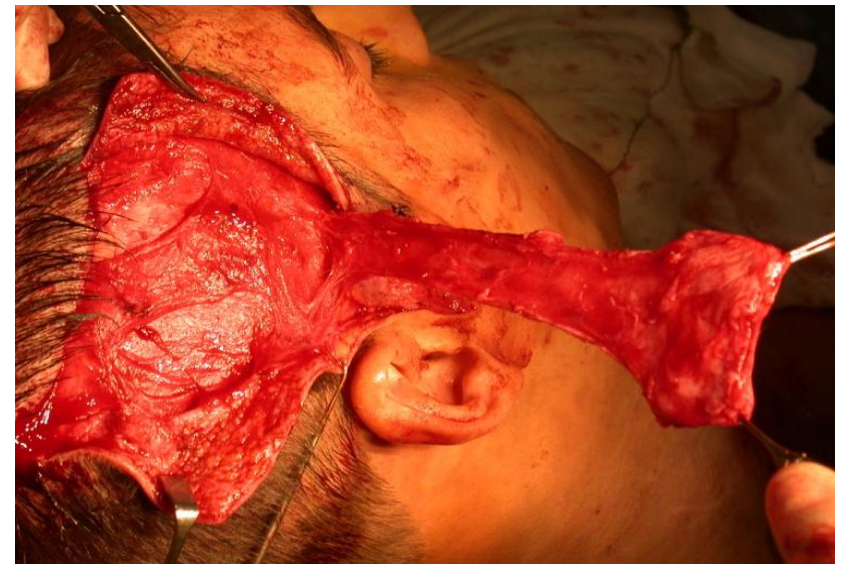

Figure 6: The rib cartilage graft prefabricated within the temporoparietal fascia is preparing to transfer. (Patient 2, intraoperative view).

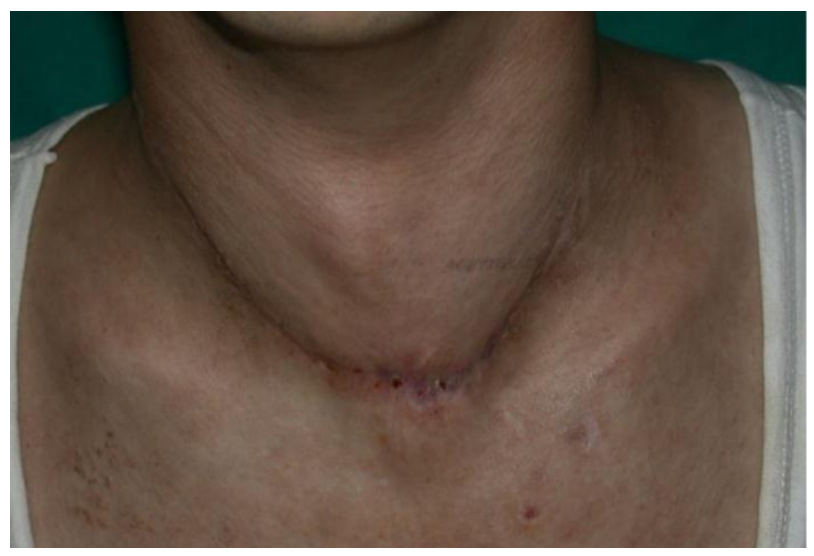

Figure 7: Postoperative view of the patient (Patient 2).

logical factors (11 patients, $19.3 \%$ ). The mean age of the tumor resection group was higher than the trauma group, $56.9 \pm 14$ years versus $30.3 \pm 6$ years, respectively. It can be commented that, head and neck tumors are mostly seen in elderly patients whereas younger people may have high rates of trauma exposure.

Among 46 tumor patients, the most common tumor type was squamous cell carcinoma (32 patients, $69.6 \%$ ). Basal cell carcinoma (9 patients, $19.6 \%$ ) and malignant melanoma (5 patients, $10.8 \%$ ) were the other histopathological diagnoses. 
All the defects were located at the head and neck region. The reconstruction sites were periorbital (37 patients, $64.9 \%$ ); malar (12 patients, $21.1 \%$ ); auricular (7 patients, $12.3 \%)$; and tracheal (1 patient, $1.7 \%$ ) region. The TPFF was used in periorbital and malar reconstruction of the tumor group patients. Five congenital and two traumatic ear deformities were reconstructed with the TPFF. A prefabricated TPFF with a cartilage graft was used to reconstruct a laryngeal defect. When necessary, additional split-thickness or full-thickness skin grafts have been used on either side of the fascia.

No major complications, including partial or total flap loss occurred. Clinical signs of infection were not observed. Alopecia at the incision site was the most common complication and was seen in 5 patients $(8.7 \%)$. It did not require further surgery and the adjacent hair growth covered the incision line. Hematoma formation occurred in 2 patients $(3.5 \%)$ due to occlusion in the drainage system. Evacuation of the hematoma and resuturing was performed. Partial skin graft losses over the flap were healed with secondary epithelialization. The injury of the frontal branch of the facial nerve was not observed in any patient.

The mean follow-up time was 20.9 months (ranging from 6 to 58 months). In each postoperative visit, patients were evaluated in terms of their operation site and wound healing. Relevant imaging studies were performed periodically in order to evaluate tumor group patients.

\section{Discussion}

The temporoparietal fascial flap has been commonly used in head and neck reconstruction. ${ }^{7,12,13}$ It has thin, broad, pliable character and also has good blood supply. Depending on the need, different tissue layers from temporal region can be included to the flap based on superficial temporal artery pedicle. Small hair bearing skin island, deep temporal fascia, and also segmental parietal bone can be raised with this flap. The TPFF and its variants can be used as free or pedicled, one or two-stage (prefabricated and prelaminated) flaps. When larger volume reconstruction is necessary, temporalis muscle can be added. If rich vascular connections between the superficial and deep temporal arteries through the loose areolar fascia below the temporal line are preserved, the temporalis muscle can be perfused by the superficial temporal vessels and reverse temporalis muscle flap can be used. ${ }^{14,15}$ When compared other regional scalp flaps such as scalp and forehead flaps it has minimal donor site morbidity. ${ }^{10}$
It can be used as a pedicled flap in the reconstruction of the orbital, mid-facial, oral, auricular, mandibular, and mastoid regions. Its thin nature makes this flap a good choice in microsurgical reconstruction of the extremities. ${ }^{16}$ The TPFF can also be used in reconstruction of the oral cavity. ${ }^{17,18}$ Prefabrication of the TPPF with skin grafts and cartilage tissue gives chance for reconstruction of nasal lining and nasal dorsum. ${ }^{18}$ In our study, we used prefabrication method in reconstruction of tracheal defect. The microsurgical transfer of the free prefabricated TPFF was performed.

Lai et al used the TPPF for orbit and periorbital region reconstruction. ${ }^{7}$ The flap may even extend to the medial canthal region and can be used in reconstruction of the eyelids. ${ }^{7}$ Obliteration of orbital cavities or skull base with fascial flap is controversial and pedicled temporalis muscle flap is considered may be a good alternative to this flap.

The TPFF is an excellent flap for coverage of exposed bone or cartilage. Acikel et al reported a case whose alar margins and atrophic nasal skin were restored in one session by primary conchal cartilage grafts, a free temporoparietal fascial flap, and a full-thickness supraclavicular skin graft. ${ }^{19}$

The temporoparietal fascial flap has been commonly used for coverage of the auricular framework in primary and secondary cases. ${ }^{3}$ It supplies thin coverage for auricular frame and leaves inconspicuous donor site scar. Park used expanded TPPF for total auricular reconstruction. ${ }^{3,12}$ In our study, this flap was used in reconstruction of seven ear deformities. In patients with congenital ear deformity, the main reconstruction process was completed with the coverage of the auricular frame with the TPFF and skin graft. In our traumatic ear deformities, the exposed auricular cartilages were saved in a single stage operation.

Fabrizio et al, repaired pharyngocutaneous fistula by using fasciocutaneous island flap from the left temporoparietal region based on the parietal branch of the superficial temporalis artery. ${ }^{13}$ In our study, the tracheal reconstruction with the TPFF is a good example of distant coverage of the defects.

Tissue expansion may be used in facial defect reconstruction and the superficial temporal vessels can be used as a vascular carrier in flap prefabrication. After the first stage TPPF operation postauricular, mastoid regions, and cervicofacial skin can be expanded. These expanded flaps are good choice for difficult facial reconstructions. ${ }^{20}$

The superficial course of the superficial temporal artery toward the vertex allows the TPFF to be elevated as a hair-bearing flap. The hair-bearing TPFF 
can be used in eyebrow, mustache or scalp's hair reconstruction. ${ }^{6,21}$

Hematoma / seroma formation, wound healing problems, alopecia, partial or total flap failure can be encountered among the complications of the TPFF surgery. Venous insufficiency is more common than arterial insufficiency. This problem can be prevented by preserving more soft tissue around the pedicle. The torsion of the pedicle and kinking in the tunnel may end up with partial or total flap loss. A special attention must be paid when transferring the flap. Incisional alopecia over the temporal site is the most common complication and it can be prevented by subfollicular dissection. We experienced alopecia at the incision site in 5 patients. This may reduce patient satisfaction. Eyebrow elevation, eyebrow ptosis are other complications that surgeon can be faced with. ${ }^{16}$

Its pliability, thinness, acceptance skin graft on both sides, high vascularity, wide range of axis of rotation, minimal donor site morbidity are some features of the of the TPFF. These advantages make this flap a good option in head and neck reconstruction. The possibility of using it as a composite flap with calvarium or hair-bearing skin and prefabrication options, provide a wider spectrum in reconstruction of composite tissue defects.

\section{Conclusion}

In conclusion, the anatomy of the temporal region as well as the temporoparietal fascia should be well known by reconstructive surgeons. This high vascularized and reliable flap has many advantages and provides reconstruction of a variety of defects.

\section{Conflict of Interest}

The authors have declared that no conflict of interest exists.

\section{References}

1. Brent B, Upton J, Acland RD, et al. Experience with the temporoparietal fascial free flap. Plast Reconstr Surg. 1985; 76: 177-88.

2. Cheney ML, Varvares MA, Nadol JBJr. The temporoparietal fascial flap in head and neck reconstruction. Arch Otolaryngol Head Neck Surg. 1993; 119: 618-23.

3. Panje WR, Morris MR. The temporoparietal fascia flap in head and neck reconstruction. Ear Nose Throat J. 1991; 70: 311-7.

4. Brent B, Byrd HS. Secondary ear reconstruction with cartilage grafts covered by axial, random, and free flaps of temporoparietal fascia. Plast Reconstr Surg. 1983; 72(2): 141-52.

5. Abul-Hassan HS, von Drasek Ascher G, Acland RD. Surgical anatomy and blood supply of the fascial layers of the temporal region. Plast Reconstr Surg. 1986; 77: 17-23.

6. Kim JC, Hadlock T, Varvares MA, Cheney ML. Hair-bearing temporoparietal fascial flap reconstruction of upper lip and scalp defects. Arch Facial Plast Surg. 2001; 3(3):170-7.

7. Lai A, Cheney ML. Temporoparietal fascial flap in orbital reconstruction.. Arch Facial Plast Surg. 2000; 2(3):196-201.
8. Byrd HS, et al. Temporoparietal (superficial temporal artery) fascial flap. In: Grabb's Encyclopedia of Flaps, 3rd ed. US: Lippincott Williams \& Wilkins; 2008: 19-22.

9. Berkowitz KB, et al. Head and neck anatomy, face and scalp. In: Gray's Anatomy The anatomical basis of clinical practice, 39th ed. Elsevier Churchill Livingstone; 2005: 497-519.

10. Mathes SJ, Nahai F. Regional Flaps: Anatomy and Basic Techniques Head and Neck Section. In: Reconstructive Surgery Principles, Anatomy, Technique Volume 1. Churchill Livingstone 1997: 367-85.

11. Greenstein B, Strauch B. Reconstruction. In: Carl E Silver, ed. Atlas of Head and Neck Surgery. Churchill Livingstone; 1999: 63.

12. Park C, Mun HY. Use of an expanded temporoparietal fascial flap technique for total auricular reconstruction. Plast Reconstr Surg. 2006; 118(2): 374-82.

13. Fabrizio T, Donati V, Nava M. Repair of the pharyngocutaneous fistula with a fasciocutaneous island flap pedicled on the superficial temporalis artery. Plast Reconstr Surg. 2000; 106(7): 1573-6.

14. Chen CT, Robinson JBJr, Rohrich RJ, Ansari M. The blood supply of the reverse temporalis muscle flap: anatomic study and clinical implications. Plast Reconstr Surg. 1999; 103(4): 1181-88.

15. Menderes A, Yilmaz M, Vayvada H, Demirdover C, Barutcu A. Reverse temporalis muscle flap for the reconstruction of orbital exenteration defects. Ann Plast Surg. 2002; 48(5): 521-6.

16. Rose EH and Norris MS. The versatile temporoparietal fascial flap: Adaptability to a variety of composite defects. Plast. Reconstr. Surg. 1990; 85: 224.

17. Nayak VK, Deschler DG. Pedicled temporoparietal fascial flap reconstruction of select intraoral defects. Laryngoscope. 2004; 114(9): 1545-8.

18. Upton J, Ferraro N, Healy G, Khouri R, Merrell C: The use of prefabricated fascial flaps for lining of the oral and nasal cavities. Plast Reconstr Surg. 1994; 94: 573.

19. Acikel C, Bayram I, Eren F, Celikoz B. Free temporoparietal fascial flaps and full-thickness skin grafts in aesthetic restoration of the nose.. Aesthetic Plast Surg. 2002; 26(6):416-8.

20. Liu Y, Jiao P, Tan X, Zhu S. Reconstruction of facial defects using prefabricated expanded flaps carried by temporoparietal fascia flaps. Plast Reconstr Surg. 2009; 123(2): 556-61.

21. Navarro-Ceballos R, Bastarrachea RA. Clinical applications of temporoparietal hair-bearing flaps for male pattern baldness and mustache formation. Aesthetic Plast Surg. 1991; 15(4):343-8. 